Check for updates

Cite this: J. Mater. Chem. A, 2021, 9, 19894

\section{A Ni or Co single atom anchored conjugated microporous polymer for high-performance photocatalytic hydrogen evolution $\dagger$}

\author{
Chen Yang, ${ }^{a}$ Zhonghua Cheng, ${ }^{a}$ Giorgio Divitini, (D) ${ }^{\text {b }}$ Cheng Qian, ${ }^{a}$ Bo Hou (D) ${ }^{c}$ \\ and Yaozu Liao (iD *a
}

Received 27th March 2021

Accepted 24th May 2021

DOI: $10.1039 / \mathrm{d} 1 \mathrm{ta02547c}$

rsc.li/materials-a

\begin{abstract}
The fabrication of single atom photocatalysts via a simple pathway is a crucial challenge to enable efficient production of hydrogen. Herein, we demonstrate a gaseous diffusion strategy to construct single atom photocatalysts by using the intrinsic nanopore of a pyridyl-functionalized conjugated microporous polymer (PCMP) to host nickel (Ni) or cobalt (Co) atoms. Comprehensive microscopy and spectroscopy characterization studies were carried out to understand the morphology and structure variations of the $\mathrm{Ni}$ or Co single atom anchored PCMP as photocatalysts. The experimental results show that Ni or Co is present as single atom anchored with pyridyl nitrogen, which prominently alters the electronic structures of the PCMP and delocalizes the charge density of the metal atom to promote proton adsorption. The outcome of the single atom anchoring substantially reduces the energy barrier of photocatalytic water splitting. As a result, $\mathrm{Ni}$ or $\mathrm{Co}$ single atom photocatalysts exhibit efficient hydrogen evolution performance with a rate of $1.72 \mathrm{mmol} \mathrm{g}^{-1} \mathrm{~h}^{-1}$ (AQE $=2.05 \%$ at $420 \mathrm{~nm}$ ) under visible-light irradiation compared to pure PCMP. Moreover, the photocatalysts show excellent stability with negligible decreases in the rate of hydrogen evolution upon long-term cycling (25 h). Our findings offer a rational way for the engineering of single atom photocatalysts for energy and environment-related applications.
\end{abstract}

\section{Introduction}

Photocatalytic water splitting is considered as a promising green route to produce hydrogen, which will be one of the most critical energy sources in the future. ${ }^{1}$ Among the developed photocatalysts, noble metal-based catalysts such as platinum, palladium, and gold are the most effective co-catalysts for hydrogen production. ${ }^{2-4}$ However, the cost of utilizing noble metals would limit their commercialization and massproduction due to their scarcity and high cost. Therefore, the development of cost-effective hydrogen evolution photocatalysts with high activities is of great significance to enable the hydrogen economy.

Conjugated polymers (CPs) consisting of naturally abundant elements such as $\mathrm{C}, \mathrm{H}$, and $\mathrm{N}$ represent emerging low-cost semiconducting materials for photocatalytic water splitting. ${ }^{5-8}$ CPs offer a unique scope for precisely tuning their

\footnotetext{
${ }^{a}$ State Key Laboratory for Modification of Chemical Fibers and Polymer Materials, College of Materials Science and Engineering, Donghua University, Shanghai 201620, China.E-mail: yzliao@dhu.edu.cn

${ }^{b}$ Department of Materials Science and Metallurgy, University of Cambridge, 27 Charles Babbage Road, Cambridge, CB3 OFS, UK

${ }^{c}$ School of Physics and Astronomy, Cardiff University, Cardiff, CF24 3AA, Wales, UK

$\dagger$ Electronic supplementary information (ESI) available. See DOI: $10.1039 / \mathrm{d} 1 \mathrm{ta} 02547 \mathrm{c}$
}

optoelectronic properties by facile molecular engineering. ${ }^{\mathbf{9 - 1 1}}$ Several CPs with various structures and morphologies have been developed for visible-light-driven water splitting hydrogen evolution. $^{12-17}$ As a new family of CPs, recently, conjugated microporous polymers (CMPs) with extended $\pi$-conjugation and intrinsic nanoporosity have drawn attention particularly. ${ }^{18-20}$ Given the distinct topological properties and functionalities of CMPs, other emerging applications such as gas uptake and separation, ${ }^{21-24}$ energy storage ${ }^{25-27}$ heterogeneous catalysis, ${ }^{28-30}$ and sensing ${ }^{31}$ can also be envisaged. Despite extensive research over the past few years worldwide, the practical implementation of such CMP photocatalysts is still limited by their slow charge separation and instability. ${ }^{32,33}$ Moreover, traditional syntheses of CMPs as photocatalysts usually involve expensive metal catalysts and energy consumption. Therefore, facile green synthesis of low-cost CMPs with suitable functionalities and optoelectronic structures for efficient visible-lightdriven water splitting remains a challenge. More recently, semiconductors doped with nonprecious transition metals as single-atom catalysts (SACs) have emerged as a new frontier in photocatalysis science because SACs have maximum atomutilization efficiency and excellent catalytic reaction activity. CMPs provide a unique platform for the preparation of SAC photocatalysts owing to their rich nanopores, high surface area, adjustable energy gap, and tunable functionality. ${ }^{34-36}$ Unfortunately, the examples of single-atom doped CMPs for 
photocatalytic hydrogen evolution applications have not been achieved yet. To achieve single-atom doped CMP photocatalysts, various chemical routes can be potentially explored by modifying the methods applied on organic and inorganic porous materials, including photochemistry, wet impregnation, $\mathrm{NH}_{3}$ treatment, and atomic layer deposition techniques. ${ }^{37-42}$ However, these approaches are often energy-intensive, involving expensive equipment as well as extreme preparation conditions. Therefore, it is highly desirable to develop an alternative method to prepare nonprecious single-atom metal-doped CMPs for photocatalytic hydrogen evolution.

In this study, we propose a facile gas diffusion strategy to prepare novel single-atom catalysts using a pyridylfunctionalized conjugated microporous polymer (PCMP) as a precursor and nickel (Ni) and cobalt (Co) as dopants for efficient photocatalytic hydrogen evolution. First, the PCMP is synthesized via a modified Chichibabin reaction according to the method previously reported, ${ }^{43}$ offering a green low-cost approach to prepare conjugated microporous polymers as new photocatalysts. Then, nickelocene or cobaltocene is successfully trapped into the PCMP precursor via evaporation and adsorption. We found that the active sites and band edge positions of the PCMP semiconductor can be well-modulated by the content of metal dosages and metal species. Owing to the presence of isolated $\mathrm{Co}-\mathrm{N}$ and pyridyl $\mathrm{N}$ active sites, the resultant PCMP photocatalysts with highly dispersed Co single-atoms exhibit a much-enhanced hydrogen evolution rate and long-term durability. Such a Co single atom anchored PCMP exhibits $>200 \%$ improvement in hydrogen evolution compared to pure PCMP under visible light irradiation. Our findings provide a versatile route toward constructing a new class of CMP photocatalysts with isolated single-atom metal centres for water splitting hydrogen generation.

\section{Results and discussion}

The synthetic routes to the PCMP and corresponding metal ( $\mathrm{Ni}$ or Co) anchored PCMP are shown in Fig. 1. By refluxing at $115{ }^{\circ} \mathrm{C}$ for $24 \mathrm{~h}$, the Chichibabin reaction between 1,3-diacetylbenzene ( $m$-DAB) and $2,2^{\prime}$-bipyridyl-5, $5^{\prime}$-dialdehyde (BPD) in the presence of ammonium acetate and pyridine gave a yellowish PCMP powder with a good yield of $80 \%$ (Fig. 1a).

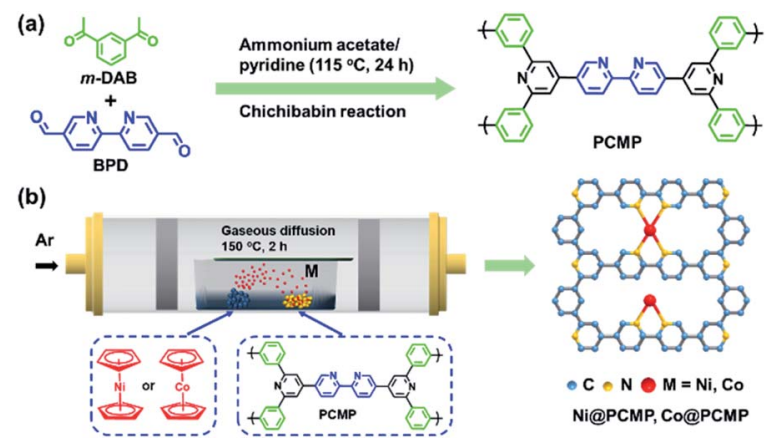

Fig. 1 Synthetic routes of (a) PCMP and (b) single atom photocatalysts i.e. Ni@PCMP, Co@PCMP.
This approach provides a green avenue to synthesize the PCMP, which is usually difficult to obtain via the metal-catalyzed crosscoupling of pyridine monomers (Table $\mathrm{S} 1 \dagger$ ). ${ }^{43}$ The polymerization mechanism of the PCMP is shown in Fig. S1. $\dagger$ Verification of the structure of the PCMP was achieved by Fourier transform infrared (FT-IR) and solid-state ${ }^{13} \mathrm{C}$ cross-polarization with magic angle spinning nuclear magnetic resonance $\left({ }^{13} \mathrm{C} \mathrm{CP} / \mathrm{MAS}\right.$ NMR) spectral analyses. For comparison, the FT-IR spectra of the building blocks of $m$-DAB and BPD are provided. After the reaction, the band at $\sim 1680 \mathrm{~cm}^{-1}$ assigned to the carbonyl group of $m$-DAB and BPD disappeared in the PCMP, ${ }^{44}$ whilst the band at $\sim 1600 \mathrm{~cm}^{-1}$ assigned to the pyridyl group became much stronger (Fig. S2a $\dagger$ ), ${ }^{45}$ manifesting successful Chichibabin coupling. The solid-state ${ }^{13} \mathrm{C}$ CP/MAS NMR spectrum of the PCMP (Fig. S2b $\dagger$ ) exhibits four primary resonances at $\sim 154$, $\sim 147, \sim 135$, and $\sim 120$ ppm owing to the ortho-, para- and metasubstituted carbon of the pyridine unit and the carbon connecting two pyridine units, ${ }^{46}$ respectively, identifying the chemical structure of the PCMP as shown in Fig. 1a. $\mathrm{N}_{2}$ adsorption/desorption isotherms indicate the microporosity of the PCMP with a specific surface area of $324 \mathrm{~m}^{2} \mathrm{~g}^{-1}$ and an average pore size of $0.7 \mathrm{~nm}$ (Fig. S3†). Therefore, the pyridyl functionality and nanopore of the PCMP offer the possibility of anchoring single atom metals via nanoconfinement and coordination interaction. To realize the idea, a general gaseous diffusion of nickelocene or cobaltocene was applied to dope a trace amount of isolated Ni or Co active centers on the PCMP support. Upon heating, nickelocene or cobaltocene powder was first sublimed into a gas phase, and then diffused and trapped into the PCMP support. Compared to traditional methods (Table S1 $\dagger$ ), our strategy gave a novel transition metal single atom anchored PCMP i.e. xM@PCMP (where $x$ represents the dosage of nickelocene or cobaltocene metal precursors in mass related to the PCMP support and $\mathrm{M}$ represents $\mathrm{Ni}$ or $\mathrm{Co}$ ) (Fig. 1b).

Upon Ni or Co doping, the as-prepared Ni@PCMP and Co@PCMP show decreased specific surface areas from $324 \mathrm{~m}^{2}$ $\mathrm{g}^{-1}$ to 100 and $154 \mathrm{~m}^{2} \mathrm{~g}^{-1}$, respectively (Fig. S3a $\dagger$ ). The inductively coupled plasma mass spectrometry (ICP-MS) analysis and full survey X-ray photoelectronic spectral (XPS) results indicate that $\mathrm{Ni}$ and Co elements have been successfully anchored on the PCMP via the gaseous diffusion strategy (Table S2 and Fig. S4a, S5a $\dagger$ ). The ICP-MS results show that the concentrations of Co (0.06-0.32 wt\%) present in Co@PCMPs is lower than that of $\mathrm{Ni}$ (2.2-4.26 wt\%) present in Ni@PCMPs at the same activation temperature. The Ni@PCMPs and Co@PCMPs show the characteristic FT-IR spectra of the pure PCMP (Fig. S6a and c†). X-ray diffraction (XRD) patterns confirm amorphous structures of Ni@PCMPs and Co@PCMPs (Fig. S6b and d广े). Scanning electron microscope (SEM) and transmission electron microscope (TEM) images show that the agglomerates of the PCMP consist of nanoparticles with diameters of $\sim 70 \mathrm{~nm}$ (Fig. S7 $\dagger$ ). Upon doping with either Ni or Co, the nanoparticles of the PCMP became more aggregated and tended to form sheet-like morphologies (Fig. 2a, b and S8a, b†). The medium-resolution aberration-corrected high-angle annular dark-field scanning transmission electron microscopy (AC-HAADF-STEM) image of 


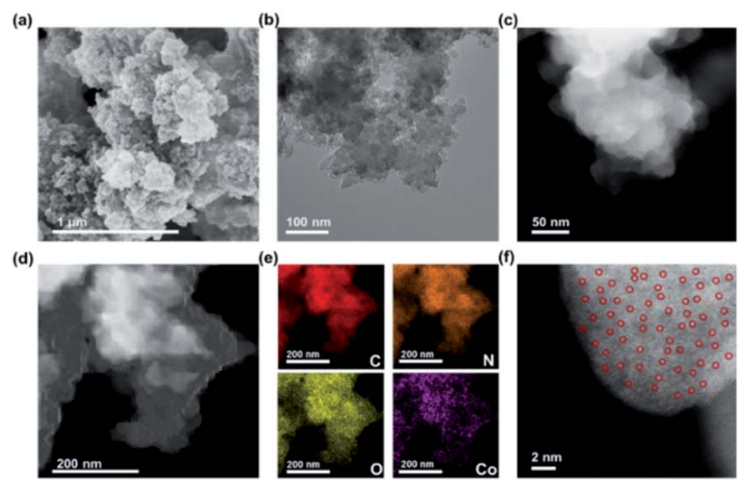

Fig. 2 (a) SEM, (b) TEM, (c) medium and (d) low-resolution ACHAADF-STEM, (e) elemental maps, and (f) high-resolution AC-HAADFSTEM images of $30 \%$ Co (aPCMP.

a typical 30\%Co@PCMP displays no Co species particles (Fig. 2c), suggesting that Co atoms are homogeneously dispersed and coordinated with $\mathrm{N}$ atoms of the PCMP. Elemental mapping images of 30\%Co@PCMP further revealed that the Co element is highly dispersed and well distributed in a polymeric framework (Fig. $2 \mathrm{~d}$ and e). The high-resolution ACHAADF-STEM image in Fig. $2 \mathrm{f}$ clearly presents many bright dots distributed over a large area, with no clusters or small particles being observed, signifying that Co is dispersed at the atomic scale. ${ }^{47}$ Although the Ni element is also homogenously distributed in the PCMP, both Ni single atoms and Ni nanoclusters are clearly present (Fig. S8c-f $\mathrm{f}^{\dagger}$ ). These results indicate that Co single atoms are easily stabilized by the PCMP compared to Ni owing to the higher diffusion barriers, electronegativity and low surface free energy. ${ }^{48,49}$

C1s, N1s, Ni2p and Co2p core-level XPS spectra were also obtained to analyze the structure of $x \mathrm{M} @ \mathrm{PCMP}$. The C1s corelevel XPS spectra confirm the formation of the desired structures (Fig. 1b), displaying three peaks at $\sim 284.6, \sim 285.4$ and $\sim 287.1 \mathrm{eV}$, which can be attributed to aryl $-\mathrm{C}=\mathrm{C}-,-\mathrm{C}=\mathrm{N}-$ and
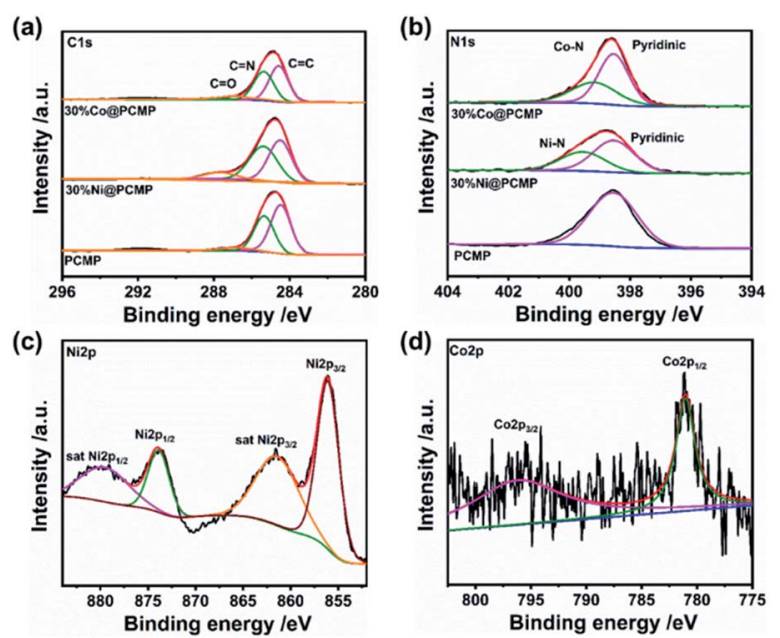

Fig. 3 (a) C1s and (b) N1s, (c) Ni2p, and (d) Co2p core-level XPS spectra of the PCMP, 30\%Ni@PCMP, and 30\%Co@PCMP.
$-\mathrm{C}=\mathrm{O}$ - bonds (Fig. 3a), respectively. The PCMP exhibits a single N1s XPS peak at $\sim 398.6 \mathrm{eV}$ owing to the pyridinic group (Fig. 3b). ${ }^{50,51}$ Besides, 30\%Ni@PCMP and 30\%Co@PCMP display two additional peaks at 399.5 and $399.2 \mathrm{eV}$ due to $\mathrm{Ni}-\mathrm{N}$ and $\mathrm{Co}-\mathrm{N}$ bonds, respectively, indicating $\mathrm{Ni}-\mathrm{N}$ and $\mathrm{Co}-\mathrm{N}$ coordination. ${ }^{41-53}$ For 30\%Ni@PCMP, it can be seen that the Ni2p XPS peak splits into two satellite Ni2 $\mathrm{p}_{1 / 2}$ and Ni2 $\mathrm{p}_{3 / 2}$ spin orbits due to oxidized Ni (Fig. 3c). ${ }^{54,55}$ For 30\%Co@PCMP, it can be seen that the Co2p XPS peak splits into two Co2 $\mathrm{p}_{1 / 2}$ and $\mathrm{Co}_{2} \mathrm{p}_{3 / 2}$ spin orbits at 781.0 and $796.2 \mathrm{eV}$ (Fig. $3 \mathrm{~d}$ ), implying the formation of $\mathrm{Co}-\mathrm{N}^{56,57}$ By altering the dosage of $\mathrm{Ni}$ and $\mathrm{Co}$ metals, the obtained samples also present similar bond information (Fig. S4 and $\mathrm{S} 5 \dagger$ ). Such a Ni-N and $\mathrm{Co}-\mathrm{N}$ coordination is beneficial to the hydrogen production performance of PCMP since these coordinations are able to accept photons, separating the electron-hole pairs easily. ${ }^{58,59}$

To gain more insights into the relationship between the structure and photocatalytic activity, ultraviolet-visible (UV-vis) diffuse reflectance spectra were examined to investigate the band gap $\left(E_{\mathrm{g}}\right)$ of Ni@PCMPs and Co@PCMPs (Fig. S9†). It can be seen from Fig. 4a, compared with PCMP $(\sim 500 \mathrm{~nm})$ and $30 \%$ Co@PCMP ( $\sim 500 \mathrm{~nm}), 30 \% \mathrm{Ni@PCMP} \mathrm{(600-700} \mathrm{nm)} \mathrm{has}$ a wider fundamental absorption range. According to the Kubelka-Munk function, ${ }^{60} E_{\mathrm{g}}$ is determined from the absorption spectra using the following equation:

$$
\alpha h \nu=A\left(h \nu-E_{\mathrm{g}}\right) n / 2
$$

where $\alpha, h, \nu, E_{\mathrm{g}}$, and $A$ represent the optical absorption coefficient, Planck constant, frequency of the incident photon, band gap and a constant depending on electron-hole mobility, respectively. The value of $n$ is 1 . The values of $\alpha$ and $A$ can be obtained according to the following equations:

$$
\begin{gathered}
\alpha=A(1-R)^{2} / 2 R \\
A=-\lg (R)
\end{gathered}
$$
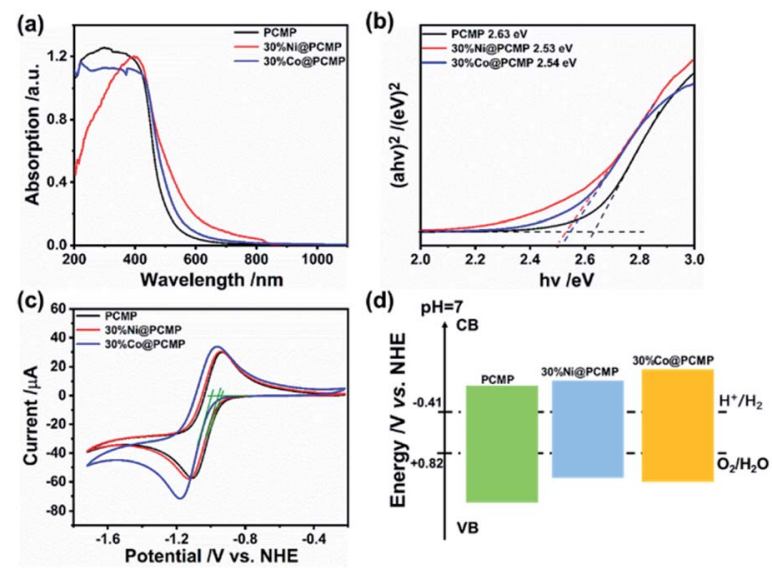

Fig. 4 (a) UV-vis spectra, (b) Tauc plots, (c) CV curves, and (d) schematic illustration of the electronic band structures of the PCMP, 30\% Ni@PCMP and 30\%Co@PCMP. 
where $R$ is the reflectance. According to eqn (1)-(3), the $E_{\mathrm{g}}$ values of the as-synthesized samples were calculated. The results demonstrate that $E_{\mathrm{g}}$ of PCMP, 30\%Ni@PCMP and 30\% Co@PCMP is 2.63, 2.53, and $2.54 \mathrm{eV}$, respectively (Fig. $4 \mathrm{~b}$ and Table 1), indicating that all catalysts have enough band gap for photocatalytic water splitting. ${ }^{61-63}$ Meanwhile, the $E_{\mathrm{g}}$ values of the Ni or Co single atom anchored PCMP can be regulated by decreasing and then increasing the dosage of $\mathrm{Ni}$ and Co from $10 \%$ to $50 \%$ (Tables S3 and S4 $\dagger$ ), respectively. Both Ni@PCMPs and Co@PCMPs exhibit the narrowest $E_{\mathrm{g}}$ when $30 \%$ of metal dosage is applied. Upon single atom anchoring, Ni or Co atoms tend to be embedded into the PCMP network and act as bridges to facilitate the charge carrier transfer owing to the effects of the conduction band offset, explaining that both $30 \% \mathrm{Ni@PCMP}$ and 30\%Co@PCMP show reduced band gaps compared with the pure PCMP. It is widely recognized that the reduced band gaps are beneficial for the light absorption ability of photocatalysts, which is crucial for improving the photocatalytic performance.

Cyclic voltammetry (CV) measurements were further conducted to identify the conduction band (CB) positions of the Ni@PCMP and Co@PCMP samples (Fig. 4c, d and S10, S11†). The valence band (VB) positions were derived by adding the $E_{g}$ to the $E_{\mathrm{CB}}$ value. ${ }^{64}$ In Table 1, the different $E_{\mathrm{CB}}$ and $E_{\mathrm{VB}}$ energy levels of PCMP, 30\%Ni@PCMP and 30\%Co@PCMP are presented, where $E_{\mathrm{Cв}}$ positions are much higher than the potentials of $\mathrm{H}^{+} / \mathrm{H}_{2}(-0.41 \mathrm{eV}, \mathrm{pH}=7)$. Since the redox potentials for proton reduction and water oxidation at $\mathrm{pH}=7$ are -0.41 and $+0.82 \mathrm{eV}$ versus the NHE level, the electronic band structures of the three samples are well-positioned for visible-light-driven hydrogen generation. It is found that the $E_{\mathrm{CB}}$ values of the samples follow the sequence: $30 \%$ Co@PCMP (more negative than $-1.00 \mathrm{eV})>30 \% \mathrm{Ni}$ @PCMP $(-0.95 \mathrm{eV})>\operatorname{PCMP}(-0.93 \mathrm{eV})$. With increasing the dosage of metals from 0 to $50 \%, 30 \%$ Ni@PCMP and 30\%Co@PCMP exhibit the most negative $E_{\mathrm{CB}}$ positions, respectively. These results indicate that $30 \%$ Co@PCMP has the strongest reduction ability. ${ }^{65}$

The hydrogen evolution experiments were performed using photocatalyst powders suspended in water under visible light irradiation $(\lambda>420 \mathrm{~nm}$, Xe lamp), where triethanolamine (TEOA) as the hole sacrificial agent and Pt (3 wt\%) as the cocatalyst were added. ${ }^{66-69}$ The hydrogen evolution performance quantitatively characterized by gas chromatography (GC) is shown in Fig. 5a and S12. $\dagger$ Both Ni@PCMPs and Co@PCMPs exhibit the highest hydrogen evolution rates when $30 \%$ of metal dosage is used. Encouragingly, the photocatalytic hydrogen evolution activity of 30\%Co@PCMP was enhanced more than 200\% compared with pure PCMP. 30\%Co@PCMP exhibits much higher hydrogen evolution performance compared to
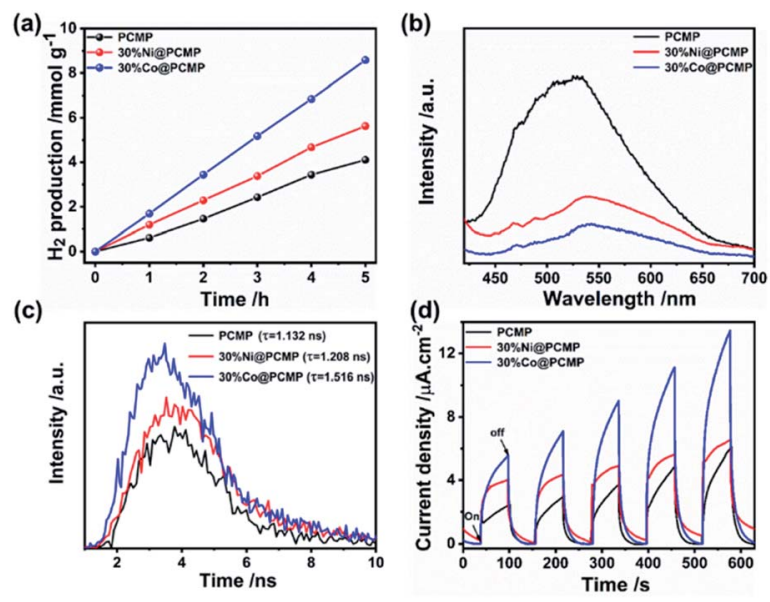

Fig. 5 (a) Time courses of hydrogen production, (b) photoluminescence emission spectra ( $\lambda_{\text {ex }}=380 \mathrm{~nm}$ ), (c) time-resolved photoluminescence decay spectra $\left(\lambda_{\mathrm{ex}}=337 \mathrm{~nm}\right)$ and $(\mathrm{d})$ time courses of photocurrent of the PCMP, 30\%NiaPCMP and 30\%Co@PCMP.

PCMP (1.72 vs. $\left.0.84 \mathrm{mmol} \mathrm{g}^{-1} \mathrm{~h}^{-1}\right)$ and 30\%Ni@PCMP (1.72 vs. $\left.0.97 \mathrm{mmol} \mathrm{g}^{-1} \mathrm{~h}^{-1}\right)$. This hydrogen evolution rate is higher than most recently reported values for conjugated polymers (such as PCMPs, CTFs, CTPs, and IEPs) and metal-modified polymers (such as PCP1e-PCP4e, P1s, Cd-TBAPy and DETPPs) (Table S5†). Compared to PCMP and 30\%Ni@PCMP (0.77\% and 1.19\%), much enhanced apparent quantum efficiency (AQE, measured at $420 \mathrm{~nm}$ ) found for 30\%Co@PCMP $(2.05 \%)$ explains the excellent hydrogen evolution performance. Meanwhile, considering that the referenced materials were synthesized via the palladium-catalyzed coupling reaction under harsh reaction conditions (high temperature, high pressure, long time complicated equipment etc.), our protocol provides a promising green route to prepare low-cost photocatalysts for water splitting. For comparison, the photocatalytic hydrogen evolution activity of the PCMP, 30\%Ni@PCMP and 30\%Co@PCMP without Pt is also provided (Fig. S13 and S14†). These results demonstrate that the hydrogen generation rate of the PCMP, $30 \% \mathrm{Ni} @ P C M P$ and 30\%Co@PCMP is 0, 196.52, and 505.27 $\mu \mathrm{mol} \mathrm{g}{ }^{-1} \mathrm{~h}^{-1}$, respectively, indicating that catalytic activity is derived from $\mathrm{Ni}$ or Co single atoms, especially Co. Considering the suitable electronic band structures of the PCMP, 30\% Ni@PCMP and 30\%Co@PCMP for water oxidation, photocatalytic oxygen evolution was further evaluated. As shown in Fig. S15, $\uparrow 30 \%$ Co@PCMP shows much higher oxygen evolution performance compared to the PCMP (48vs. $15 \mu \mathrm{mol} \mathrm{g}^{-1} \mathrm{~h}^{-1}$ ) and 30\%Ni@PCMP (48vs. $27 \mu \mathrm{mol} \mathrm{g}^{-1} \mathrm{~h}^{-1}$ ).

Table 1 Metal content, band gaps $\left(E_{\mathrm{g}}\right)$ and band positions $\left(E_{\mathrm{CB}}\right.$ and $\left.E_{\mathrm{VB}}\right)$ of the PCMP, 30\%Ni@PCMP and 30\%Co@PCMP

\begin{tabular}{lllllll}
\hline Sample & $\mathrm{Ni}(\mathrm{wt} \%)$ & $\mathrm{Co}(\mathrm{wt} \%)$ & $E_{\mathrm{g}}(\mathrm{eV})$ & $E_{\mathrm{CB}}(\mathrm{eV})$ & $E_{\mathrm{VB}}(\mathrm{eV})$ & $\mathrm{H}_{2}$ evolution rate $\left(\mathrm{mmol} \mathrm{g}^{-1} \mathrm{~h}^{-1}\right)$ \\
\hline PCMP & - & - & 2.63 & -0.93 & 1.7 & 0.83 \\
30\%Ni@PCMP & 2.97 & - & 2.53 & -0.95 & 1.58 & 0.97 \\
30\%Co@PCMP & - & 0.14 & 2.54 & -1.00 & 1.54 & 1.72
\end{tabular}


Photoluminescence (PL) and time-resolved photoluminescence decay spectra were examined to compare the charge separation efficiency among the PCMP, Ni@PCMPs and Co@PCMPs. A strong PL emission peak located at approximately $523 \mathrm{~nm}$ was observed for the PCMP (Fig. 5b), which may be attributed to the recombination radiation of photoinduced charge carriers and excitons. ${ }^{70}$ However, the position of the PL emission peaks of Ni@PCMPs ( 537 nm) and Co@PCMPs $(\sim 541 \mathrm{~nm})$ is close to that of PCMP $(\sim 523 \mathrm{~nm})$, whose intensity drastically reduced (Fig. S16 $\dagger$ ). Especially, the PL emission intensity of 30\%Ni@PCMP and 30\%Co@PCMP has been significantly reduced. Much higher charge separation efficiency was observed for 30\%Co@PCMP compared to the values observed for 30\%Ni@PCMP and PCMP (Fig. 5b), which was also confirmed from the time-resolved PL decay spectra (Fig. 5c). It is found that the average lifetime of the PCMP $(1.13 \mathrm{~ns})$ increased to $1.21 \mathrm{~ns}$ and $1.52 \mathrm{~ns}$ for 30\%Ni@PCMP and 30\%Co@PCMP, respectively. The longer lifetime is believed to be beneficial for photocarrier transfer, ${ }^{71}$ explaining the improved hydrogen evolution observed for Co@PCMP. The time courses of photocurrent of the samples indicate that the most substantial photocurrent response is found for 30\%Co@PCMP (Fig. 5d). The obtained Nyquist plots (Fig. S17†) indicate that the diameters of the semicircles of 30\%Ni@PCMP and 30\%Co@PCMP are smaller than that of the PCMP, particularly 30\%Co@PCMP, suggesting that the single atom metals contribute to photogenerated electron-hole pair separation and efficient charge transfer better. $^{72}$ The results signify that 30\%Co@PCMP is much more sensitive to visible light irradiation and effectively promotes the separation and transfer of photo generated electron-hole pairs, which is consistent with the better photocatalytic activity observed.

To further evaluate the hydrogen evolution performance, the stability of the PCMP, 30\%Ni@PCMP and 30\%Co@PCMP was further investigated upon long-term irradiation. As can be seen in Fig. 6a, steady photocatalytic hydrogen evolution is observed

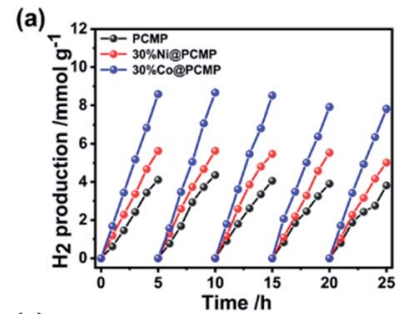

(c)

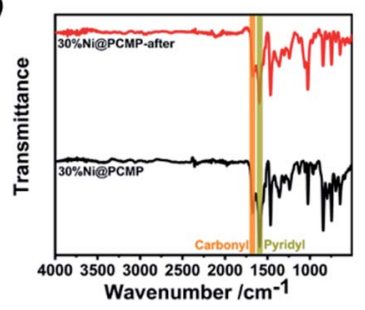

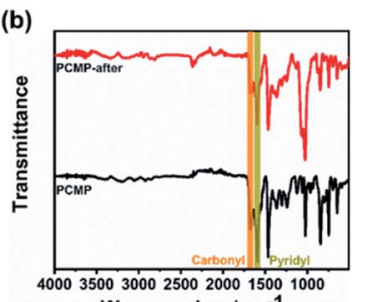

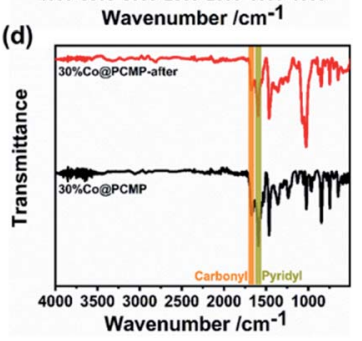

Fig. 6 Photocatalytic stability tests: (a) time courses of hydrogen production and (b)-(d) FT-IR spectra of the PCMP, 30\%Ni@PCMP, and 30\%Co@PCMP upon $25 \mathrm{~h}$ cycling. in five consecutive runs over a total of $25 \mathrm{~h}$. The SEM images show that the PCMP, 30\%Ni@PCMP and 30\%Co@PCMP retain their original morphology upon $25 \mathrm{~h}$ irradiation (Fig. S18 $\dagger$ ). XRD patterns confirm that the PCMP, 30\%Ni@PCMP and 30\% Co@PCMP are almost unchanged (Fig. S19†). The FT-IR spectral analyses show that both the functional groups and intrinsic conjugated skeletons of polymers maintain the same state upon $25 \mathrm{~h}$ irradiation (Fig. 6b-d), verifying the excellent recyclability and photocorrosion-resistance of the PCMP, 30\%Ni@PCMP and 30\%Co@PCMP. The stability was further confirmed by XPS spectral analyses (Fig. 7 and S20, S21 $\dagger$ ). Upon $25 \mathrm{~h}$ of irradiation, the main XPS peaks of all the photocatalysts show negligible changes. The C1s, N1s, Ni2p and Co2p core-level XPS spectra also show that the peaks of $-\mathrm{C}=\mathrm{C}-,-\mathrm{C}=\mathrm{N}-,-\mathrm{C}=\mathrm{O}-$, $\mathrm{Ni}-\mathrm{N}$ and $\mathrm{Co}-\mathrm{N}$ bonds retain the original position upon irradiation, further testifying the excellent stability of the asprepared photocatalysts. To testify the universality of the strategy, 30\%Fe@PCMP was also prepared using ferrocene as a precursor. The SEM, TEM and elemental mapping observations (Fig. S22 $\dagger$ ) indicate that the Fe element was successfully anchored on the PCMP. However, 30\%Fe@PCMP exhibits a slight increase in hydrogen evolution performance compared to the PCMP (0.87 vs. $0.84 \mathrm{mmol} \mathrm{g}^{-1} \mathrm{~h}^{-1}$ ) (Fig. S23†). This may be related to the instability, diffusion barriers and electronegativity of the Fe element. ${ }^{73}$

Based on the above results and discussion, a possible mechanism of photocatalytic hydrogen evolution for $x \mathrm{M} @ \mathrm{PCMP}$ is proposed in Fig. S24. $\dagger$ Photogenerated electrons $\left(\mathrm{e}^{-}\right)$jump to the $E_{\mathrm{CB}}$ level, and holes $\left(\mathrm{h}^{+}\right)$to the $E_{\mathrm{VB}}$ level. The electrons are further transferred to the surface of the PCMP for hydrogen evolution, and the holes are consumed by the sacrificial agent. A significant increase of the photocatalytic hydrogen evolution for Ni@PCMPs or Co@PCMPs can be explained as follows: (1) the $\mathrm{Ni}$ or Co single-atom content modulates the energy band position and effectively promotes
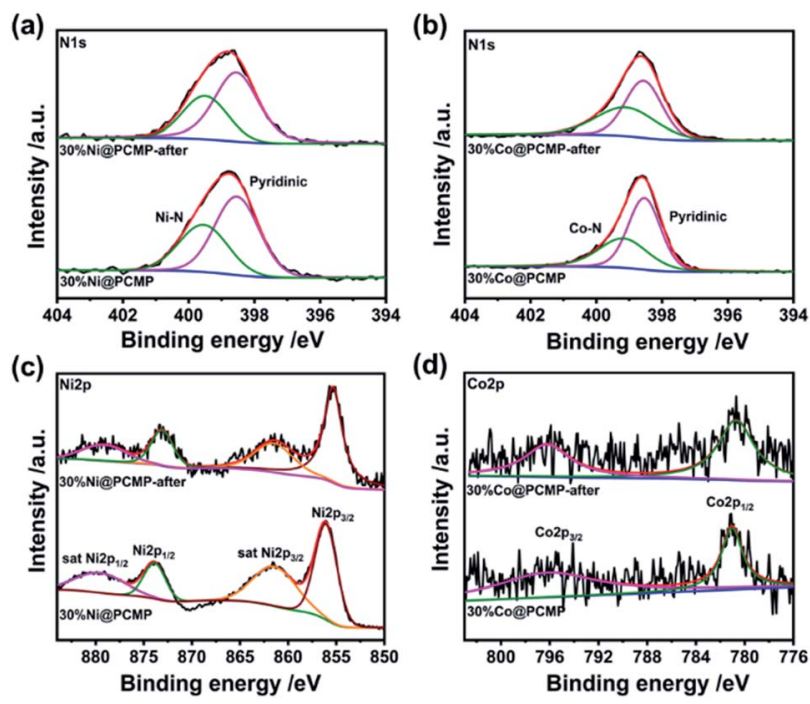

Fig. 7 (a and b) N1s, (c) Ni2p, and (d) Co2p core-level XPS spectra of the PCMP, 30\%NiaPCMP, and 30\%Co@PCMP upon $25 \mathrm{~h}$ cycling 
the separation and transfer efficiency of photo-generated carriers; (2) the interaction between $\mathrm{Ni}$ and $\mathrm{N}$ or Co and $\mathrm{N}$ atoms enhances the electron transfer, changing the electronic configuration and increasing the active sites. Overall, the enhanced photocatalytic activity observed in 30\%Co@PCMP can be attributed to the improved negative redox potential, surface-active sites, charge separation efficiency, and photocarrier transfer, as well as photo sensitivity.

\section{Conclusions}

In summary, a gaseous diffusion strategy has been successfully demonstrated for the synthesis of a $\mathrm{Ni}$ or Co single-atom anchored pyridyl-functionalized conjugated microporous polymer (PCMP). The synthesis can be conducted at a relatively low temperature $\left(150{ }^{\circ} \mathrm{C}\right)$ in a short period $(2 \mathrm{~h})$. The as-prepared Ni@PCMP and Co@PCMP photocatalysts possess suitable electronic structures for visible-light-driven hydrogen evolution activity. Especially, 30\%Co@PCMP exhibited the highest photocatalytic $\mathrm{H}_{2}$ evolution activity, and the steady photocatalytic hydrogen evolution rate reached up to $1.72 \mathrm{mmol} \mathrm{g}^{-1} \mathrm{~h}^{-1}$, which is $200 \%$ times higher than that of the pure PCMP. By selecting suitable metal contents, we believe that this work could open a versatile and facile route for designing highly efficient CMP anchored single-atom photocatalysts.

\section{Author contributions}

Y. Liao initiated and supervised the project. C. Yang designed and performed the experiments, analyzed the data, and prepared all illustrations. Z. Cheng, G. Divitini, C. Qian and B. Hou performed the SEM, TEM, STEM measurements and helped with the data analyses. All the authors wrote the manuscript and have given approval to the final version of the manuscript.

\section{Conflicts of interest}

The authors declare no competing financial interest.

\section{Acknowledgements}

This work was supported by the National Natural Science Foundation of China (52073046, 51873036 and 51673039), the Chang Jiang Scholar Program (Q2019152), the Shanghai Shuguang Program (19SG28), and the Natural Science Foundation of Shanghai (19ZR1470900). We furthermore acknowledge the support from the International Joint Laboratory for Advanced Fiber and Low-Dimension Materials (18520750400).

\section{Notes and references}

1 C. F. Shih, T. Zhang, J. Li and C. Bai, Joule, 2018, 2, 1-25.

2 H. Wei, H. Wu, K. Huang, B. Ge, J. Ma, J. Lang, D. Zu, M. Lei, Y. Yao, W. Guo and H. Wu, Chem. Sci., 2019, 10, 2830-2836. 3 W. Zhao, Y. Jiao, J. Li, L. Wu, A. Xie and W. Dong, J. Catal., 2019, 378, 42-50.

4 Y. K. Kim and H. Park, Energy Environ. Sci., 2011, 4, 685-694.
5 C. Han, P. Dong, H. Tang, P. Zheng, C. Zhang, F. Wang, F. Huang and J. Jiang, Chem. Sci., 2021, 12, 1796-1802.

6 M. Sachs, H. Cha, J. Kosco, C. M. Aitchison, L. Francas, S. Corby, C. Chiang, A. A. Wilson, R. Godin, A. FaheyWilliams, A. I. Cooper, R. S. Sprick, I. McCulloch and J. R. Durrant, J. Am. Chem. Soc., 2021, 143, 524.

7 H. Yang, H. Amari, L. Liu, C. Zhao, H. Gao, A. He, N. D. Browning, M. A. Little, R. S. Sprick and A. I. Cooper, Nanoscale, 2020, 12, 24488-24494.

8 Y. Zang, R. Wang, P. Shao, X. Feng, S. Wang, S. Zang and T. C. W. Mak, J. Mater. Chem. A, 2020, 8, 25094-25100.

9 H. Sirringhaus, Science, 1998, 280, 1741-1744.

10 L. Wang, Y. Zhang, L. Chen, H. Xu and Y. Xiong, Adv. Mater., 2018, 30, 1801955.

11 C. Yang, B. C. Ma, L. Zhang, S. Lin, S. Ghasimi, K. Landfester, K. A. I. Zhang and X. Wang, Angew. Chem., Int. Ed., 2016, 55, 9202-9206.

12 G. Li, Z. Xie, Q. Wang, X. Chen, Y. Zhang and X. Wang, Chem.-Eur. J., 2021, 27, 939-943.

13 F. Wang, W. Lei, X. Pan and Z. Ye, Nanotechnology, 2020, 31, 475406.

14 J. Xiao, X. Liu, L. Pan, C. Shi, X. Zhang and J. Zuo, ACS Catal., 2020, 10, 12256-12283.

15 Q. Yang, M. Luo, K. Liu, H. Cao and H. Yan, Appl. Catal., B, 2020, 276, 119174.

16 J. Xu, C. Yang, S. Bi, W. Wang, Y. He, D. Wu, Q. Liang, X. Wang and F. Zhang, Angew. Chem., Int. Ed., 2020, 59, 23845-23853.

17 H. Ye, Z. Wang, Z. Yang, S. Zhang, X. Gong and J. Hua, J. Mater. Chem. A, 2020, 8, 20062-20071.

18 N. B. Mckeown and P. M. Budd, Macromolecules, 2010, 43, 5163-5176.

19 J. M. Lee and A. I. Cooper, Chem. Rev., 2020, 120, 2171-2214.

20 G. Zhang, W. Ou, J. Wang, Y. Xu, D. Xu, T. Sun, S. Xiao, M. Wang, H. Li, W. Chen and C. Su, Appl. Catal., B, 2019, 245, 114-121.

21 X. Hu, H. Wang, C. F. J. Faul, J. Wen, Y. Wei, M. Zhu and Y. Liao, Chem. Eng. J., 2020, 382, 122998.

22 Z. Cheng, Z. Dai, J. Li, H. Wang, M. Huang, X. Li and Y. Liao, Chem. Eng. J., 2019, 357, 776-786.

23 Y. Liao, Z. Cheng, W. Zuo, A. Thomas and C. F. J. Faul, ACS Appl. Mater. Interfaces, 2017, 9, 38390-38400.

24 Y. Liao, J. Weber, B. M. Mills, Z. Ren and C. F. J. Faul, Macromolecules, 2016, 49, 6322-6333.

25 M. Zhang, T. Zhao, J. Dou, Z. Xu, W. Zhang, X. Chen, X. Wang and B. Zhou, ChemElectroChem, 2019, 6, 5946-5950.

26 Y. Li, W. Li, Z. Cheng, Y. He, H. Li, H. Li and Y. Liao, ChemNanoMat, 2019, 6, 58-63.

27 H. Wang, Z. Cheng, Y. Liao, J. Li, J. Weber, A. Thoams and C. F. J. Faul, Chem. Mater., 2017, 29, 4885-4893.

28 L. Sekerová, P. Březinová, T. T. Do, E. Vyskoclilova, J. Krupka, L. Cerveny, L. Havelkova, B. Bashta and J. Sedlacek, ChemCatChem, 2019, 12, 1075-1084.

29 X. Wang, L. Chen, S. Y. Chong, M. A. Little, Y. Z. Wu, W. H. Zhu, R. Clowes, Y. Yan, M. A. Zwijnenburg, R. S. Sprick and A. I. Cooper, Nat. Chem., 2018, 10, 11801189. 
30 L. Chen, Y. Yang and D. Jiang, J. Am. Chem. Soc., 2010, 132, 9138-9143.

31 X. Liu, Y. Xu and D. Jiang, J. Am. Chem. Soc., 2012, 134, 87388741.

32 R. S. Sprick, J. X. Jiang, B. Bonillo, S. J. Ren, T. Ratvijitvech, P. Gui, M. A. Zwijnenburg, D. J. Adams and A. I. Cooper, J. Am. Chem. Soc., 2015, 137, 3265-3270.

33 X. Wang, X. Zhao, W. Dong, X. Zhang, Y. Xiang, Q. Huang and H. Chen, J. Mater. Chem. A, 2019, 7, 16277-16284.

34 A. Pimachev, R. D. Nielsen and Y. Dahnovsky, Chem. Phys., 2020, 26, 14480-14488.

35 X. Zhang, H. Liu, P. An, Y. Shi, J. Han, Z. Yang, C. Long, J. Guo, S. Zhao, K. Zhao, H. Yin, L. Zheng, B. Zhang, X. Liu, L. Zhang, G. Li and Z. Tang, Sci. Adv., 2020, 6, 24824.

36 H. Wang, B. Hou, Y. Yang, Q. Chen, M. Zhu, A. Thoams and Y. Liao, Small, 2018, 14, 1803232.

37 Z. Qian and K. A. I. Zhang, Sol. RRL, 2021, 5, 2000489.

38 Q. Zhao, J. Sun, S. Li, C. Huang, W. Yao, W. Chen, T. Zeng, Q. Wu and Q. Xu, ACS Catal., 2018, 8, 11863-11874.

39 L. Zeng, C. Dai, B. Liu and C. Xue, J. Mater. Chem. A, 2019, 7, 24217-24221.

40 Y. Cao, S. Chen, Q. Luo, H. Yan, Y. Lin, W. Liu, L. Cao, J. Lu, J. Yang, T. Yao and S. Wei, Angew. Chem., Int. Ed., 2017, 56, 12191-12196.

41 M. Piernavieja-Hermida, Z. Lu, A. White, K. B. Low, T. Wu, J. F. Elam, Z. Wu and Y. Lei, Nanoscale, 2016, 8, 15348-15356.

42 T. He, S. Chen, B. Ni, Y. Gong, Z. Wu, L. Song, L. Gu, W. Hu and X. Wang, Angew. Chem., Int. Ed., 2018, 57, 3493-3498.

43 Z. Cheng, L. Wang, Y. He, X. Chen, X. Wu, H. Xu, Y. Liao and M. Zhu, Polym. Chem., 2020, 11, 3393-3397.

44 L. Zou, D. Feng, T. F. Liu, Y. P. Chen, S. Fordham, S. Yuan, J. Tian and H. C. Zhou, Chem. Commun., 2015, 51, 40054008.

45 H. Cao, H. Huang, Z. Chen, B. Karadeniz, J. Lv and R. Cao, ACS Appl. Mater. Interfaces, 2017, 9, 5231-5236.

46 Y. He, Z. Cheng, H. Zuo, C. Yan and Y. Liao, ChemElectroChem, 2020, 7, 959-966.

47 Y. Xiong, W. Sun, P. Xin, W. Chen, X. Zheng, W. Yan, L. Zheng, J. Dong, J. Zhang, D. Wang and Y. Li, Adv. Mater., 2020, 32, 2000896.

48 Y. Huang, C. An, Q. Zhang, L. Zang, H. Shao, Y. Liu, Y. Zhang, H. Yuan, C. Wang and Y. Wang, Nano Energy, 2021, 80, 105535.

49 J. Huang, C. Zhou, Z. Chu, X. Liu and X. Duan, Phys. Chem. Chem. Phys., 2021, 23, 1868-1873.

50 Z. Sheng, L. Shao, J. Chen, W. Bao, F. Wang and X. Xia, ACS Nano, 2011, 5, 4350-4358.
51 A. Maetz, L. Delmotte, G. Moussa, J. Dentzer, S. Knopf and C. M. Ghimbeu, Green Chem., 2017, 19, 2266-2274.

52 X. Ning, Y. Sun, H. Fu, X. Qu, Z. Xu and S. Zheng, Chemosphere, 2020, 241, 124978.

53 F. Mao, P. F. Liu, P. Yang, J. Gu and H. Yang, Chem. Commun., 2020, 56, 7495-7498.

54 Y. Zhou, D. Lin, X. Ye and M. Zhu, J. Alloys Compd., 2020, 839, 155691.

55 L. Li, J. Xu and B. Su, Appl. Surf. Sci., 2020, 524, 146154.

56 H. Gong, X. Hao, H. Li and Z. Jin, J. Colloid Interface Sci., 2021, 585, 217-228.

57 Q. Zhao, W. Yao, C. Huang, Q. Wu and Q. Xu, ACS Appl. Mater. Interfaces, 2017, 9, 42734-42741.

58 Y. Xing, J. Ku, W. Fu, L. Wang and H. Chen, Chem. Eng. J., 2020, 395, 125149.

59 X. Li, H. Zhang, Y. Liu, X. Duan and S. Wang, Chem. Eng. J., 2020, 390, 124634.

60 P. Sivakarthik, V. Thangara and M. Parthibavarman, J. Mater. Sci.: Mater. Electron., 2017, 28, 5990-5996.

61 L. Fang, X. Wang, Y. Li, P. Liu, Y. Wang, H. Zeng and H. Yang, Appl. Catal., B, 2017, 200, 578-584.

62 L. Li, Z. Deng, L. Yu, Z. Lin, W. Wang and G. Yang, Nano Energy, 2016, 27, 103-113.

63 Q. Xiang, J. Yu and M. Jaroniec, Nanoscale, 2011, 3, 36703678.

64 B. Hou, D. Parker, G. P. Kissling, J. A. Jones, D. Cherns and D. J. Fermín, J. Phys. Chem. C, 2013, 117, 6814-6820.

65 J. Wang, P. Guo, M. Dou, J. Wang, Y. Cheng, P. Jonsson and Z. Zhao, RSC Adv., 2014, 4, 51008-51015.

66 Q. Shang, N. Liu, D. You, D. Q. Cheng, G. Liao and Z. Pan, J. Mater. Chem. C, 2021, 9, 238-248.

67 G. Shu, Y. Li, W. Zhuang, J. Jiang and F. Wang, Appl. Catal., $B, 2019,261,118230$.

68 Y. Wang, C. Zheng, M. Liu, W. Wei, J. Gao, Y. Zhang and P. Deng, Chem. Commun., 2021, 57, 3857-3860.

69 L. Luo, Z. Gong, J. Ma, K. Wang, H. Zhu, K. Li, L. Xiong, X. Guo and J. Tang, Appl. Catal., B, 2021, 284, 119742.

70 J. Li, H. Huang, P. Liu, X. Song, D. Mei, Y. Tang, X. Wang and C. Zhong, J. Catal., 2019, 375, 351-360.

71 W. Lin, M. H. Elsayed, J. Jayakumar, L. Ting, C. Chang, M. E. Ahmed, W. Wang, C. Chung, C. Lu and H. Chou, Int. J. Hydrogen Energy, 2020, 45, 32072-32081.

72 J. Xie, C. Wang, N. Chen, W. Chen, J. Xu, P. Bai, B. Liu, L. Zhang and H. Wang, J. Mater. Chem. C, 2021, 9, 4378.

73 Y. Huang, C. An, Q. Zhang, L. Zhang, H. Shao, Y. Liu, Y. Zhang, H. Yuan, C. Wang and Y. Wang, Nano Energy, 2021, 80, 105535. 